\title{
Critical points at infinity, non-Gaussian saddles, and bions
}

\author{
Alireza Behtash, ${ }^{a, b}$ Gerald V. Dunne, ${ }^{a, c}$ Thomas Schäfer, ${ }^{b}$ Tin Sulejmanpasic $^{a, d}$ \\ and Mithat Ünsal ${ }^{a, b}$ \\ ${ }^{a}$ Kavli Institute for Theoretical Physics, University of California, \\ Santa Barbara, CA 93106, U.S.A. \\ ${ }^{b}$ Department of Physics, North Carolina State University, \\ Raleigh, NC 27695, U.S.A. \\ ${ }^{c}$ Department of Physics, University of Connecticut, \\ Storrs, CT 06269-3046, U.S.A. \\ ${ }^{d}$ Philippe Meyer Institute, Physics Department, École Normale Supérieure, \\ PSL Research University, \\ 24 rue Lhomond, F-75231 Paris Cedex 05, France \\ E-mail: proof.beh@gmail.com, dunne@phys.uconn.edu, tmschaef@ncsu.edu, \\ tin.sulejmanpasic@gmail.com, unsal.mithat@gmail.com
}

ABSTRACT: It has been argued that many non-perturbative phenomena in quantum mechanics (QM) and quantum field theory (QFT) are determined by complex field configurations, and that these contributions should be understood in terms of Picard-Lefschetz theory. In this work we compute the contribution from non-BPS multi-instanton configurations, such as instanton-anti-instanton $[\mathcal{I} \overline{\mathcal{I}}]$ pairs, and argue that these contributions should be interpreted as exact critical points at infinity. The Lefschetz thimbles associated with such critical points have a specific structure arising from the presence of non-Gaussian, quasi-zero mode (QZM), directions. When fermion degrees of freedom are present, as in supersymmetric theories, the effective bosonic potential can be written as the sum of a classical and a quantum potential. We show that in this case the semi-classical contribution of the critical point at infinity vanishes, but there is a non-trivial contribution that arises from its associated non-Gaussian QZM-thimble. This approach resolves several puzzles in the literature concerning the semi-classical contribution of correlated $[\mathcal{I} \overline{\mathcal{I}}]$ pairs. It has the surprising consequence that the configurations dominating the expansion of observables, and the critical points defining the Lefschetz thimble decomposition need not be the same, a feature not present in the traditional Picard-Lefschetz approach.

KEYWORDS: Nonperturbative Effects, Differential and Algebraic Geometry, Resummation ARXIV EPRINT: 1803.11533 


\section{Contents}

1 Introduction 1

2 Classical action and critical points at infinity 5

2.1 Cluster expansion 6

$\begin{array}{lll}2.2 & \text { Critical points at infinity } & 7\end{array}$

2.3 Overview of the BZJ method 7

2.4 QZM-thimble 9

3 Quantum action and bions 11

3.1 Critical points at infinity and their thimbles vs. exact bion solutions 12

$\begin{array}{lll}3.1 .1 & {[\mathcal{I} \mathcal{I}] \text { thimble integration }} & 12\end{array}$

$\begin{array}{ll}3.1 .2[\mathcal{I} \overline{\mathcal{I}}] \text { thimble integration } & 13\end{array}$

$\begin{array}{llr}3.2 & \text { Real bion amplitude vs. }[\mathcal{I} \mathcal{I}] \text { amplitude } & 16\end{array}$

$\begin{array}{lll}3.3 & \text { Complex bion amplitude versus }[\mathcal{I} \overline{\mathcal{I}}] \text { amplitude } & 18\end{array}$

$\begin{array}{llr}4 \text { Conclusions } & 19\end{array}$

$\begin{array}{ll}\text { A Fluctuation determinants } & 19\end{array}$

A.1 Fluctuation determinant for real bion saddle 20

A.2 Fluctuation determinants for real bounce and complex bion saddles 22

\section{Introduction}

The semi-classical expansion is an invaluable tool in quantum field theory and quantum mechanics $[1,2]$. However, beyond leading order, the naive semi-classical "instanton gas" expansion is typically ill-defined. The standard example of the problems that arise at higher order is that of an instanton-anti-instanton $[\mathcal{I} \overline{\mathcal{I}}]$ pair. The instanton is a real saddle point of the path integral, characterized by a "fugacity" $e^{-S_{I} / g}$. But since instantons and anti-instantons attract one another, in the instanton gas framework there is no exact saddle point in the $[\mathcal{I} \overline{\mathcal{I}}]$ sector at finite separation. The action of such a configuration continuously decreases as the two pseudo-particles get closer. On the other hand, we expect physical observables, such as quantum mechanical energies, to have a well-defined expansion in powers of the instanton fugacity. Some progress was made by Bogomolny and Zinn-Justin a long time ago [2-4], who computed higher order terms using analytic continuation, as reviewed below. However, this calculation left several conceptual and practical questions unanswered. In this paper, we resolve some of these issues, revisiting the BZJ analysis by taking advantage of recent progress in applying resurgence theory [5-14] and PicardLefschetz theory [15-21] to path integrals. 
It has become clear that the semi-classical expansion naturally lives in the complexified field space [5, 12, 17-20, 22-27]. The infinite dimensional space that appears in path integration is to be deformed into hypersurfaces in the complexified field space attached to the saddles. Such hypersurfaces (known as "Lefschetz thimbles") should be chosen to guarantee that the integration always yields a convergent result. A convenient choice of these manifolds is generated by the complexified gradient flow equations in the field space $[17,18,28]$ (as opposed to the real gradient flow equations or valley methods, cf. [29]) given by

$$
\frac{\partial \phi}{\partial u}=\frac{\delta \bar{S}[\bar{\phi}]}{\delta \bar{\phi}}
$$

Here, $\phi(x, u)$ is the (complexified) field that depends on the Euclidean space-time point $x$, and the gradient flow time $u$, and $S[\phi]$ is the holomorphic action functional. The flow equation (1.1) ensures that the real part of the action increases along the flow, while the imaginary part remains constant, and hence naturally generalizes the stationary phase approximation in ordinary finite-dimensional integrals [30, 31]. If the flow equation is initialized in the vicinity of the saddle point,${ }^{1}$ then the flow equations define a hypersurface with the two desired features: (1) the thimble contains the saddle point, and (2) the path integral converges with the fields along the thimble.

This observation implies that "unstable" saddles of the Euclidean action (i.e. critical points which are saddles rather than the minima of the action) should be treated on an equal footing with those saddles that minimize the action. Historically, this point led to some confusion, because it was not always clear how to incorporate solutions with negative fluctuation modes. In principle, Picard-Lefschetz theory provides conceptual clarity in such situations. Note also that the contribution of "unstable" saddles generically requires the integration to be performed along complex manifolds in field space.

This issue arises unavoidably at second (and higher) order in the semi-classical expansion. To describe physics at $O\left(e^{-2 S_{I} / g}\right)$, the saddles $[\mathcal{I} \mathcal{I}],[\overline{\mathcal{I}} \overline{\mathcal{I}}]$ and $[\mathcal{I} \overline{\mathcal{I}}]$ must all be treated on an equal footing. In the literature it is often asserted that instanton-anti-instanton configurations are not critical points of the action, because of the existence of a classical interaction between them of the form $V(\tau) \sim \pm \frac{1}{g} e^{-\tau}$, where $\tau$ is the quasi-zero mode direction. However, this potential has a critical point at infinity. ${ }^{2}$ In this work, by considering certain quantum mechanical (QM) systems on an Euclidean temporal circle with finite size $\beta$, we first find critical points at finite $\beta$. Upon taking $\beta \rightarrow \infty$ such saddles move to infinity. We show that the multi-instanton expansion, or cluster expansion, performed by using the Lefschetz thimbles associated with the QZM directions, resolves some important puzzles concerning the BZJ calculation of multi-instanton effects. We argue that the cluster expansion on the $\Gamma_{\mathrm{QZM}}$ thimble provides a conceptually complete framework for performing semi-classical expansions.

In our earlier studies $[12,23]$ we considered two prototypical QM examples: (i) systems with degenerate harmonic minima coupled to $N_{f}$ Grassmann valued fields (corresponding to

\footnotetext{
${ }^{1}$ The flow equation clearly cannot be initialized exactly at the saddle point, since the r.h.s. of (1.1) vanishes there.

${ }^{2}$ Interesting properties of critical points at infinity in ordinary integrals have been discussed in [30].
} 
spin $\left(\frac{1}{2}\right)^{N_{f}}$ ); and (ii) the related bosonic systems that arise after integrating out fermions, which are characterized by non-degenerate harmonic minima. In fact, integrating out the Grassmann fields exactly, one obtains an effective quantum potential (as in $\mathcal{N}=1$ supersymmetric quantum mechanics $[32,33]$ )

$$
V(x)=v_{0}(x)+p g v_{1}(x),
$$

where $v_{0}(x)$ is the classical potential, $p$ is a parameter related to $N_{f}$, and $v_{1}(x)$ is the potential generated by integrating out the fermions. Note that the second term, involving $v_{1}(x)$, is a quantum correction, proportional to the coupling constant $g$. For $p \neq 0$, we refer to the potential $V(x)$ as the effective quantum potential. In $[12,23]$ it was demonstrated that the bosonic theory governed by $V(x)$ can exhibit both real and complex bion configurations. Both of these configurations are exact solutions of the equations of motion of the quantum action, corresponding to the effective quantum potential in (1.2). In particular, the complex bion is not a solution of the ordinary (real) classical equations of motion in the inverted potential (as described in standard textbooks, see [1, 2]); rather it is an exact solution of the holomorphic classical equations of motion associated with the effective potential in (1.2).

In the works $[12,20,22-25,34,35]$ many arguments were made that indicate such complex solutions of the quantum action dominate the semi-classical analysis. But this observation, while having many merits and explaining a number of puzzles, led to several new puzzles, some of which are listed below.

- The relevant saddles are critical points of the exact effective action, which can typically be thought of as containing fermionic loop effects. However according to PicardLefschetz theory it is the classical action, rather than the quantum one, that is related to the thimble decomposition relevant for the resurgent structure.

- If the quantum corrections are promoted to the classical action (i.e. the $g$-suppressed part of the effective action is promoted to be of order unity), such saddles no longer play a role in the resurgent expansion. ${ }^{3}$

- The saddle points have a non-zero imaginary part, which is crucial for the resurgent cancellations, as well as to make sense of the real physical contributions. However, they appear to violate the intersection theorem of the thimbles and their corresponding dual thimbles.

- Generic quantum mechanical problems, with classically degenerate vacua, may still have different curvatures, resulting in quantum non-degeneracy [38]. After one loop effects are taken into account, such systems appear to again be saturated by complex saddles of one-loop actions. Unlike fermions which can be easily integrated out

\footnotetext{
${ }^{3}$ This observation was used in $[36,37]$ to avoid dealing with non-perturbative contributions entirely, by formally treating $g$-suppressed terms as order-unity terms. This procedure reshuffles the perturbation theory, effectively putting some coupling dependence into coefficients of the series expansion. With this scaling, the quantum tilt becomes classical and perturbation theory is Borel summable.
} 
exactly, the bosonic one loop action has infinitely many terms, and it is unclear how to set up a systematic treatment of such problems in the same spirit.

- Finally, this approach had no adequate treatment of the multi-instanton corrections of the problems with quantum degenerate minima (e.g. traditional double-well and sine-Gordon problems), except that they could be treated as a limiting case. While avoiding some of the difficulties encountered in the BZJ prescription this is not entirely satisfactory, as we expect that such difficulties should possibly be overcome directly by a complete Picard-Lefschetz theory of path integrals.

The main objective of this work is to resolve these discrepancies and consolidate the Picard-Lefschetz theory with the resurgence properties which relate perturbation theory to the saddles of the quantum action. In particular, we aim to explain the precise relation between critical points at infinity associated with the classical action, their Lefschetz thimbles, and the bion solutions to the quantum action. ${ }^{4}$ We show that:

1) Due to their non-Gaussian nature, the integral over the thimble attached to the critical point at infinity may not be saturated in the vicinity of the critical point. In fact, it may well be the case that this contribution vanishes completely, concealing the effect of the saddle on the physical observables. This is in sharp contrast with the Gaussian saddles which typically appear in ordinary integrals, where the dominant contribution always originates from the saddle itself.

2) Due to the effects of the Gaussian directions, the main semi-classical contribution may arise from the tail of the QZM descent cycle $\Gamma_{\mathrm{QZM}}$, which is a consequence of the non-Gaussian nature of the quasi-zero mode directions. In the cases studied here we show that the bion saddles of the one-loop (quantum) action (for $p \neq 0$ ) dominate the integration over $\Gamma_{\mathrm{QZM}}$. Note that despite the fact that the bions are not saddles of the classical action, and the genuine critical point attached to the $\Gamma_{\mathrm{QZM}}$ thimble is at infinity, it is still the bion configuration that dominates the observables, resolving the puzzles outlined above.

3) Such complex configurations, and not the critical points at infinity, provide the complex phases that lead to hidden topological angles [22], which are crucial for explaining quantum interference effects and QM supersymmetry breaking [12, 23].

We argue that these results are generic features of non-Gaussian critical points at infinity, and that this structure persists at higher orders in the semi-classical cluster expansion.

In order to understand the relation between bion amplitudes and correlated multiinstanton amplitudes, we also discuss the bion fluctuation operators, which determine the leading pre-exponential factor of the corresponding amplitudes. The fluctuation operators for bion solutions are quite different from the familiar fluctuation operators that appear in the context of instantons $[1,2]$. The typical fluctuation operator encountered in instanton

\footnotetext{
${ }^{4}$ Indeed, it is likely the case that generic critical points of the path integral are of this type, including QFT applications.
} 
problems is a Schrödinger operator with a single-well potential, for example the PöschlTeller potential as in (2.6) below. For real bions, the fluctuation operator involves a doublePöschl-Teller potential, see (A.11) and figure 5. For complex bions, the fluctuation operator contains even more exotic complex potentials. (For periodic bosonic potentials, the complex bion fluctuation potentials can even be singular [12, 23].) For both real and complex bions, the associated fluctuation operators possess parametrically small eigenvalues that correspond to non-Gaussian directions. When this soft mode is carefully treated, there is perfect agreement between the results of the instanton gas analysis and the bion analysis.

Our work builds on earlier studies devoted to the role of complex saddle points, for example Brezin et al. [39], Balian, Parisi and Voros [40], Richard and Rouet [41], Lapedes and Mottola [42], Millard [43], and Balitsky and Yung [34]. Complexified classical solutions and Lefschetz thimbles have also been very recently studied by Nekrasov using techniques and ideas from the Bethe/gauge correspondence [27].

\section{Classical action and critical points at infinity}

Consider the classical bosonic Euclidean path integral

$$
\begin{aligned}
Z_{\mathrm{bos}} & =\int D x(t) e^{-\frac{1}{g} S[x(t)]}=\int D x(t) e^{-\frac{1}{g} \int d t\left(\frac{1}{2} \dot{x}^{2}+V_{\mathrm{bos}}(x)\right)}, \\
V_{\mathrm{bos}}(x) & =\frac{1}{2}\left(W^{\prime}(x)\right)^{2} .
\end{aligned}
$$

There are instanton and anti-instanton saddle points, which are (real) solutions of

$$
\dot{x}= \pm W^{\prime}(x) .
$$

In this non-perturbative normalization, the first order BPS equation and its solution are independent of the coupling constant $g$. We concentrate on the Sine-Gordon (SG) system, but a similar analysis can be done for the double-well system extensively studied in [12, 23]. The superpotential and the instanton solution are, respectively, given by

$$
W(x)=4 \cos \left(\frac{x}{2}\right) \quad \Rightarrow \quad x_{I}(t)=4 \arctan \left(\exp \left[t-t_{c}\right]\right)
$$

and the corresponding instanton action is

$$
S_{I}=\frac{8}{g} .
$$

Here, $t_{c} \in \mathbb{R}$ is the position modulus, a bosonic zero mode of the instanton solution. The instanton amplitude is given by

$$
\mathcal{I} \equiv \xi=J_{t_{c}} e^{-S_{I}}\left[\frac{\hat{\operatorname{det} \mathcal{M}_{I}}}{\operatorname{det} \mathcal{M}_{0}}\right]^{-\frac{1}{2}} P_{I}(g),
$$

where $J_{t_{c}}=\sqrt{S_{I} /(2 \pi)}$ is the Jacobian associated with the bosonic zero mode, and $\mathcal{M}_{I}=-\frac{d^{2}}{d t^{2}}+\left.V^{\prime \prime}(x)\right|_{x=x_{I}(t)}$ is the quadratic fluctuation operator in the background of the instanton. It has the familiar Pöschl-Teller form

$$
\mathcal{M}_{I}=-\frac{d^{2}}{d t^{2}}+1-2 \operatorname{sech}^{2}\left(t-t_{c}\right)
$$


whose only exact zero mode is given by $\dot{x}_{I}(t)$. The "hat" on det $\mathcal{M}_{I}$ indicates that the zero mode has to be removed, and $\operatorname{det} \mathcal{M}_{0}$ is a normalization factor, which we take to be the corresponding free fluctuation operator. $P_{I}(g)$ denotes the perturbative expansion around the instanton.

The determinant of the instanton fluctuation operator can be computed using the Gel'fand-Yaglom (GY) method [1, 2, 44-50], summarized in appendix A. For the instanton in the $\mathrm{SG}$ model the determinant ratio is a pure number

$$
\frac{\hat{\operatorname{det}} \mathcal{M}_{I}}{\operatorname{det} \mathcal{M}_{0}}=\frac{1}{4} .
$$

In section 3 we will also need the fluctuation determinants about more general saddle configurations such as the real and complex bions. These are quite different from the instanton fluctuation determinants, and are computed in appendix A, using a variant of the GY method [47, 49, 51, 52]. The resulting determinant, with zero mode removed, can be simply expressed in terms of the action of the saddle configuration and the asymptotic behavior of the associated zero mode.

\subsection{Cluster expansion}

The partition function can be expressed as a cluster (virial) expansion for an interacting gas of instantons $[1,2]$. Assuming that there is a gap between the ground state and the first excited state, in the $\beta \rightarrow \infty$ limit, we can write $Z$ as

$$
Z=e^{-\beta E_{0} P_{0}(g)}\left(1+\frac{\xi}{1 !} \int d \tau_{1}+\frac{\xi^{2}}{2 !} \int d \tau_{1} d \tau_{2} e^{-V_{12}}+\frac{\xi^{3}}{3 !} \int d \tau_{1} d \tau_{2} d \tau_{3} e^{-V_{123}}+\ldots\right) .
$$

In these integrals, $\tau_{i}$ denotes the position of $i$ th instanton event, and $V_{i j k \ldots}$ denotes a manybody interaction. Neglecting the interaction between instantons, the sum exponentiates and we obtain the dilute instanton gas correction to the vacuum energy as

$$
\begin{aligned}
Z_{\text {dilute }} & =e^{-\beta E_{0} P_{0}(g)}\left(\sum_{n=0}^{\infty} \frac{\xi^{n}}{n !} \int d \tau_{1} \ldots d \tau_{n}\right) \\
& =e^{-\beta E_{0} P_{0}(g)}\left(\sum_{n=0}^{\infty} \frac{\xi^{n}\left(\int d \tau_{1}\right)^{n}}{n !}\right)=e^{-\beta E_{0} P_{0}(g)} e^{\beta \xi}=e^{-\beta\left(E_{0} P_{0}(g)-\xi\right)}
\end{aligned}
$$

Note that the instanton-induced term provides a negative contribution to the ground state energy. In fact, this argument suffices to show that any real saddle contribution to the ground state energy, in the absence of a $\theta$-angle or Berry phase, is universally negative. As was noted in [12, 20,23], this observation is at odds with supersymmetry.

The interaction terms $V_{12}, V_{123}, \ldots$ are functions of the separation between the instantons. The two-body interaction can be written as $V_{12}=V_{12}\left(\tau_{1}-\tau_{2}\right)$ and the three-body term is $V_{123}:=V_{12}\left(\tau_{1}-\tau_{2}\right)+V_{23}\left(\tau_{2}-\tau_{3}\right)+V_{31}\left(\tau_{3}-\tau_{1}\right)$, where the genuine three-body interactions were neglected. Hence, using the relative coordinates $\tau_{i j}=\tau_{i}-\tau_{j}$ in each term of (2.8), one of the integrals yields a factor of $\beta$ :

$$
Z=e^{-\beta E_{0} P_{0}(g)}\left(1+\frac{\xi}{1 !} \beta+\frac{\xi^{2}}{2 !} \beta \int d \tau_{12} e^{-V_{12}\left(\tau_{12}\right)}+\frac{\xi^{3}}{3 !} \beta \int d \tau_{12} d \tau_{23} e^{-V_{123}}+\ldots\right) .
$$


The physical meaning of this factorization is the following: each instanton has one exact zero mode, related to its position. When we have $n$ instantons, due to the interaction between them, $(n-1)$ of these zero modes turn into quasi-zero modes (QZM), while one of them remains an exact zero mode, related to the center of mass. The integration over the exact zero mode gives a factor of $\beta$. On the other hand, the remaining $(n-1)$-dimensional integral is structurally very interesting, and is at the heart of the instanton expansion. In particular, the integral $\beta \int d^{n-1} \tau$ behaves as a polynomial in $\beta$ with coefficients depending on the coupling constant $g$. It has the form $\beta^{n}(\#)+\beta^{n-1}(\#)+\ldots+\beta^{1}(\#)$ that contains maximally extensive and sub-extensive terms in $\beta$. This structure is indicative of the presence of a critical point at infinity.

\subsection{Critical points at infinity}

At finite separation, there is an interaction between two instantons of the form

$$
V_{12}(\tau)= \pm \frac{A}{g} e^{-\tau} \quad \tau \equiv \tau_{12}, \quad A=32,
$$

where $\tau$ is the QZM direction, and $A=32$ with our normalization convention. The interaction is repulsive for an instanton-instanton pair, and attractive for an instanton/antiinstanton pair. As a result there is no exact saddle point at any finite separation for an $[\mathcal{I} \overline{\mathcal{I}}]$ pair in the instanton gas picture. However, at $\tau=\infty$ there is no interaction between pairs of pseudo-particles, and the configuration is indeed a critical point. Below, we show that the second order terms in the semi-classical cluster expansion generate, for an $[\mathcal{I} \overline{\mathcal{I}}]$ pair, the contribution

$$
\frac{\xi^{2}}{2 !} \int_{\Gamma} d \tau_{1} d \tau_{2} e^{-V_{12}}=\frac{\beta^{2}[\mathcal{I}][\overline{\mathcal{I}}]}{2 !}+\frac{\beta\left([\mathcal{I} \overline{\mathcal{I}}]_{ \pm}\right)^{1}}{1 !} .
$$

Here, we have performed the integral over the QZM-thimble of the critical point at infinity. The first part of this expression (maximally extensive in $\beta$ ) is of course the uncorrelated (non-interacting) dilute instanton gas contribution, and the subextensive $O(\beta)$ term is the leading term in the correlated $[\mathcal{I} \overline{\mathcal{I}}]_{ \pm}$contribution. In the cluster expansion, the terms having a form of $\beta^{2}[\mathcal{I}][\overline{\mathcal{I}}] / 2$ ! exponentiate and give a contribution of order $\beta e^{-S_{I}}$ to $\beta E_{0}$, while the summation and exponentiation of the terms of the type $\beta\left([\mathcal{I} \overline{\mathcal{I}}]_{ \pm}\right)^{1} / 1$ ! contribute at order $\beta e^{-2 S_{I}}$ to $\beta E_{0}$. The critical point at infinity and its QZM-thimble captures both types of contributions.

\subsection{Overview of the BZJ method}

We begin by reviewing the analysis of Bogomolny and Zinn-Justin [2-4]. Their result is clearly correct - as it has been checked against numerical results and the WKB method - but it raises some conceptual and computational questions that we will resolve below using recent advances in resurgence and Picard-Lefschetz theory.

The second order term in the cluster expansion of the partition function, coming from an $[\mathcal{I} \overline{\mathcal{I}}]$ pair, is

$$
Z_{2}=\xi^{2} \int_{0}^{\infty} d \tau e^{\frac{A}{g} e^{-\tau}}
$$


The above integral is divergent because the integrand in the upper limit does not go to zero. This is a benign divergence, which appears in any virial expansion. The solution is that we have to subtract the uncorrelated term, which exponentiates to the dilute instanton gas result, and then proceed to compute the correlated remainder, being a genuine instanton/anti-instanton effect. We write Virial 2 , using the " $-1+1$ trick", see e.g. [53]:

$$
Z_{2}=\xi^{2} \int_{0}^{\infty} d \tau\left(e^{\frac{A}{g} e^{-\tau}}-1+1\right)
$$

The " +1 " term leads to the appearance of $\frac{\xi^{2}}{2 !}\left(\int d \tau_{1}\right)^{2}$ in $(2.8)$, and accounts for the leading order semi-classical expansion which captures the effect of non-interacting instantons in the dilute instanton gas approximation. The correlated instanton/anti-instanton amplitude is

$$
[\mathcal{I} \overline{\mathcal{I}}]=\xi^{2} \int_{0}^{\infty} d \tau\left(e^{\frac{A}{g} e^{-\tau}}-1\right)
$$

This integral is convergent, but it is dominated by the regime $\tau \rightarrow 0$ where the notion of an instanton/anti-instanton pair does not make sense.

To evaluate this integral Bogomolny and Zinn-Justin proposed to analytically continue the coupling constant as $g \rightarrow-g$ [2-4]. By doing so one obtains a repulsive potential between the instanton and anti-instanton. Next, upon integrating by parts, the integral reduces to

$$
\begin{aligned}
{[\mathcal{I} \overline{\mathcal{I}}] } & \rightarrow-\frac{A}{g} \int_{0}^{\infty} d \tau e^{-\frac{A}{g} e^{-\tau}} e^{-\tau} \tau \\
& =-\gamma-\log \left(\frac{A}{g}\right)-\Gamma\left(0, \frac{A}{g}\right) \\
& =-\gamma-\log \left(\frac{A}{g}\right)-e^{-A / g}\left(\frac{g}{A}+O\left(g^{2}\right)\right) .
\end{aligned}
$$

BZJ drop the exponentially small terms in this expression, coming from $\Gamma\left(0, \frac{A}{g}\right)$, and then continue back to the positive coupling constant, $-g \rightarrow+g$, where one obtains the result

$$
[\mathcal{I} \overline{\mathcal{I}}]_{ \pm}=\mp i \pi-\gamma-\log \left(\frac{A}{g}\right)+\ldots
$$

Here, the sign ambiguity of the imaginary part depends on whether one analytically continues back in the upper or lower complex $g$ half-plane, respectively. As is well-known [2-4], this ambiguity in the result of the correlated $[\mathcal{I} \overline{\mathcal{I}}]_{ \pm}$amplitude cancels exactly the ambiguity that arises by resuming the perturbation theory, which, likewise, needs to be defined by analytically continuing the coupling constant into the complex plane.

A problem with this analysis is that it is not clear why it is possible to drop terms in (2.16) which are exponentially small when $\Re(g)<0$, but which become exponentially large $\sim e^{+A / g}$ when analytically continued back to $\Re(g)>0$. In the next section we show that if one properly treats critical points at infinity via the Lefschetz thimble decomposition, such issues are resolved. 


\subsection{QZM-thimble}

The thimble approach to the cluster expansion, based on Picard-Lefschetz theory, provides a conceptually cleaner and more systematic way of computing multi-instanton contributions. We consider the quantum mechanical system with periodic boundary conditions on a temporal circle of length $\beta$. The amplitude for the correlated instanton-anti-instanton pair is

$$
[\mathcal{I} \overline{\mathcal{I}}]=\frac{1}{2} \int_{0}^{\beta} d \tau e^{\frac{A}{g}\left(e^{-\tau}+e^{-(\beta-\tau)}\right)}-\beta / 2 .
$$

The modified instanton interaction reflects the periodicity of the temporal box. ${ }^{5}$ Notice that since the above integral counts the $[\mathcal{I} \overline{\mathcal{I}}]$ configuration twice (that is, integration from 0 to $\beta / 2$ is the same as from $\beta / 2$ to $\beta$ ), we have included an extra factor $1 / 2$.

The exponent of the integrand in (2.18) has a critical point, which corresponds to an exact instanton/anti-instanton solution [42]. The precise form of this solution is not relevant for our argument. ${ }^{6}$ The $[\mathcal{I} \overline{\mathcal{I}}]$ action

$$
S(\tau)=-\frac{A}{g}\left(e^{-\tau}+e^{-(\beta-\tau)}\right)
$$

correctly captures the action in the QZM direction up to higher order terms in $e^{-\tau}$. The critical point of $S(\tau)$ is easily seen to be $\tau_{0}=\beta / 2$ even though there are other saddles as well, see e.g. [42], at $\tau_{n}=\beta / 2+i \pi n$. We now transform $\tau=\tau_{0}+\xi$. However, it is immediately observed that along the original integration cycle (i.e. $\mathbb{R}$ ) the action decreases away from the critical point $\xi=0$. This just reflects the fact that the instantonanti-instanton pair attracts for real separations $\tau$ (i.e. the critical point at infinity is an "unstable" saddle in the traditional sense). The Picard-Lefschetz theory, however, the contribution of the saddle should be evaluated along the "downward flow", where the action always increases, thus ensuring convergence. Such downward flow emanates from $\tau=\tau_{0}$ directly into the imaginary $\tau$-direction, which is sketched in figure 1 . The downward flow from the saddle at $\tau_{0}$ connects to the saddles $\tau_{ \pm 1}$, meaning that we are on a Stokes line. This scenario agrees with what one expects from perturbation theory. To move off the Stokes line, we analytically continue $g \rightarrow g e^{i \theta}$. For $\theta$ being small and positive, the picture in figure 1 is obtained. For $\theta$ small and negative, the picture in figure 1 is flipped about the vertical axis.

Now it is clear that the downward flow, or the Lefschetz thimble, through the saddle at $\tau=\tau_{0}$ consists of three parts; namely

$$
\Gamma_{\mathrm{QZM}}^{\theta=0^{ \pm}}=\gamma_{1}^{ \pm} \cup \gamma_{2}^{ \pm} \cup \gamma_{3}^{ \pm}
$$

\footnotetext{
${ }^{5}$ More precisely, the exponent leading to the interaction between the instanton and anti-instanton must be periodic under shift symmetry, $\tau \rightarrow \tau+\beta$, and is thus given by $\frac{A}{g} \sum_{n=-\infty}^{\infty} e^{-|\tau+n \beta|}$. Except for the $n=0,-1$ terms that are indeed captured in (2.18), the rest gives sub-leading contributions in $e^{-\beta}$ and are henceforth ignored.

${ }^{6}$ Such solutions obviously exist, however, whenever there are two neighboring classical minima. To see this, consider a particle moving in the inverted classical potential (e.g. inverted double-well). We seek a solution which interpolates from the vicinity of one classical vacuum to another (i.e. one peak of the inverted potential to the other). These solutions exist and show an oscillatory behavior between the two peaks, with a period exactly equal to $\beta$.
} 


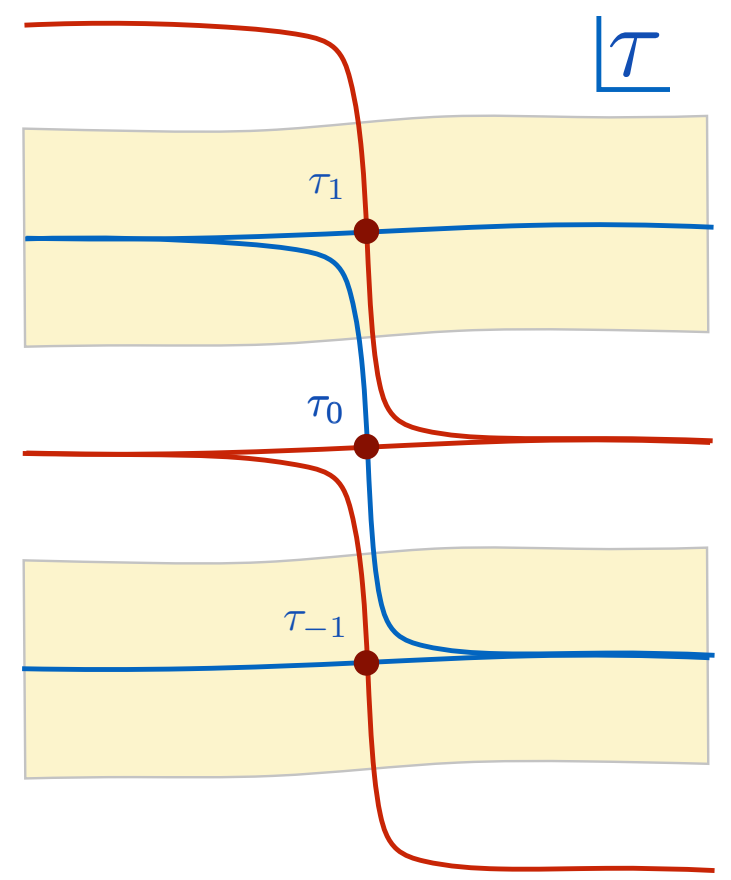

Figure 1. The Lefschetz thimbles for the $\mathcal{I} \overline{\mathcal{I}}$ saddle, showing the downward flows (blue curves) connecting $\tau_{0}$ to $\tau_{ \pm 1}$ when $g \rightarrow g e^{i \theta}$ with $\theta \rightarrow 0^{+}$. The red curves are the corresponding upward flows. The directions are flipped about the imaginary axis for $\theta \rightarrow 0^{-}$.

where the segments are

$$
\gamma_{1}^{ \pm}: \xi \in(-\infty \pm i \pi, \pm i \pi), \quad \gamma_{2}^{ \pm}: \xi \in[ \pm i \pi, \mp i \pi], \quad \gamma_{3}^{ \pm}: \xi \in(\mp i \pi, \infty \mp i \pi) .
$$

We therefore define

$$
[\mathcal{I} \overline{\mathcal{I}}]_{ \pm}=\lim _{\beta \rightarrow \infty} \frac{1}{2}\left(\int_{\cup_{i} \gamma_{i}^{ \pm}} d \xi e^{-S(\xi)}-\beta\right)
$$

Evaluating the integral along $\gamma_{2}^{ \pm}$in the limit $\beta \rightarrow \infty$ gives $\mp i \pi$. Now, it is left to compute the integral over $\gamma_{1}^{ \pm} \cup \gamma_{3}^{ \pm}$. This can be concisely written as

$$
\int_{\gamma_{1}^{ \pm} \cup \gamma_{3}^{ \pm}} d \xi e^{-S(\xi)}=\int_{-\infty}^{\infty} d \xi e^{-\frac{2 A}{g} e^{-\beta / 2} \cosh (\xi)}=2 K_{0}\left(\frac{2 A}{g} e^{-\beta / 2}\right)
$$

where $K_{0}(z)$ is a modified Bessel function of the second type. Taking $\beta \rightarrow \infty$, we can use the asymptotic behavior of $K_{0}(z)$

$$
K_{0}\left(\frac{2 A}{g} e^{-\beta / 2}\right) \rightarrow-\gamma-\log \left(\frac{A}{g}\right)+\beta / 2 .
$$

The amplitude (2.22) finally takes the form

$$
[\mathcal{I} \overline{\mathcal{I}}]_{ \pm}=\mp i \pi-\gamma-\log \left(\frac{A}{g}\right)+\ldots
$$


Clearly, this construction does not run into the conceptual difficulty mentioned in the previous section. It also clarifies that in path integration the field space (and specifically the quasi-zero mode direction) must be complexified in order to properly define the semiclassical expansion. Finally, it shows that the Lefschetz thimble associated with a critical point at infinity (not necessarily the critical point per se), yields the dominant contribution. We will see that the final point becomes more relevant once fermions are included. In that case, the critical point at infinity does not contribute at all, and the entire contribution comes from a non-Gaussian thimble.

\section{Quantum action and bions}

In this section we analyze the role of critical points at infinity in quantum mechanical models containing fermions. We consider the Euclidean path integral

$$
Z=\int D x(t) e^{-\frac{1}{g} S[x(t)]}=\int D x(t) e^{-\frac{1}{g} \int d t\left(\frac{1}{2} \dot{x}^{2}+V(x)\right)},
$$

where the bosonic potential is (recall that $W(x)=4 \cos \left(\frac{x}{2}\right)$ for the SG system)

$$
V(x)=\frac{1}{2}\left(W^{\prime}(x)\right)^{2}+\frac{p g}{2} W^{\prime \prime}(x) .
$$

The important feature of the action in (3.1) is that in addition to having a purely classical part, it has a quantum piece that is of the form

$$
V(x)=v_{0}(x)+p g v_{1}(x),
$$

where the term $p g v_{1} \equiv \frac{p g}{2} W^{\prime \prime}(x)$ arises from integrating out the fermionic degrees of freedom. ${ }^{7}$ We refer to $V(x)$ as the effective quantum potential.

For $p= \pm 1$, the quantum potential corresponds to the bosonic and fermionic sectors of supersymmetric QM. For other positive or negative integers, the quantum potential is related to quasi-exactly solvable systems. This can be seen by integrating out $N_{f}$ fermionic fields in the action

$$
S=\frac{1}{g} \int d t\left(\frac{1}{2} \dot{x}^{2}+\frac{1}{2}\left(W^{\prime}\right)^{2}+\frac{1}{2}\left(\bar{\psi}_{i} \dot{\psi}_{i}-\dot{\overline{\psi_{i}}} \psi_{i}\right)+\frac{1}{2} W^{\prime \prime}\left[\bar{\psi}_{i}, \psi_{i}\right]\right), \quad i=1, \ldots, N_{f} .
$$

The system given in (3.1) with the potential function (3.2) has exact solutions of the equations of motions associated with the quantum potential [12, 23]

$$
+\frac{d^{2} z}{d t^{2}}=\frac{\partial V}{\partial z} \quad \text { or } \quad \frac{d^{2} z}{d t^{2}}=W^{\prime} W^{\prime \prime}+\frac{p g}{2} W^{\prime \prime \prime} .
$$

These exact solutions inlcude the bounce as well as real and complex bions. The explicit form of the solutions is summarized in appendix A. Unlike instantons, the exact solutions of the equations of motion involve $g$, and have a characteristic size $\ln (A / g)$, which can be interpreted as the separation of instantons-anti-instantons constituents.

\footnotetext{
${ }^{7}$ If the second term is a classical tilt, i.e. $p g \sim O(1)$, of the potential, then complex field configurations still exist, but perturbation theory is Borel summable, see [37].
} 
It is important to note that the classical solutions, the leading saddle points coming from the classical action, are still given by instantons. At second order in the cluster expansion, two instantons at infinite separation correspond to a genuine critical point at infinity. In this section we explain the precise relation between these classical saddle points at infinity and the exact bion solutions of the quantum action.

\subsection{Critical points at infinity and their thimbles vs. exact bion solutions}

Consider the quantum system on a temporal circle with size $\beta$, as in the $p=0$ case. The presence of fermions, or equivalently, the existence of the quantum term in the potential, modifies the interaction between instantons. We denote the interaction potential between two instantons by $\mathcal{V}_{+}(z)$, and the potential between an instanton and an anti-instanton by $\mathcal{V}_{-}(z)$ :

$$
\mathcal{V}_{ \pm}(\tau)= \pm \frac{A}{g}\left(e^{-\tau}+e^{-(\beta-\tau)}\right)+p \tau
$$

The interaction between instantons has both classical and quantum terms. The classical bosonic interaction is repulsive for correlated $[\mathcal{I} \mathcal{I}]$ pairs and attractive for $[\mathcal{I} \overline{\mathcal{I}}]$ pairs. On the other hand, the fermion zero mode (or quantum) induced potential, $\frac{p g}{2} W^{\prime \prime}(x)$, leads to an attractive interaction for both $[\mathcal{I} \mathcal{I}]$ and $[\mathcal{I} \overline{\mathcal{I}}]$ pairs.

\subsection{1 $[\mathcal{I} \mathcal{I}]$ thimble integration}

We discuss first the slightly simpler case of an $[\mathcal{I I}]$ pair configuration. This configuration is a critical point at infinity, which means that $\tau_{0}=\beta / 2$ goes to infinity as $\beta \rightarrow \infty$. In contrast to a Gaussian critical point, the contribution from this critical point vanishes as $\beta \rightarrow \infty$,

$$
\lim _{\beta \rightarrow \infty} e^{-\frac{2 A}{g}\left(e^{-\beta / 2}\right)} e^{-p \beta / 2}=0 .
$$

This does not mean that the contribution of the thimble vanishes. Indeed, the exact integration over $\Gamma_{\mathrm{QZM}}^{\theta=0^{ \pm}}$in the $\beta \rightarrow \infty$ limit gives

$$
I_{+}(p, g) \equiv \int_{\Gamma_{\mathrm{QZM}}^{\theta=0 \pm}} d \tau e^{-\frac{A}{g}\left(e^{-\tau}+e^{-(\beta-\tau)}\right)} e^{-p \tau}=\left(\frac{g}{A}\right)^{p} \Gamma(p)
$$

The $[\mathcal{I I}]$ amplitude, derived from integrating over the associated QZM-thimble, is

$$
\begin{aligned}
{[\mathcal{I} \mathcal{I}] } & =I_{+}(p, g) \times[\mathcal{I}]^{2} \\
& =\left(\frac{g}{A}\right)^{p} \Gamma(p) \times \frac{S_{I}}{2 \pi}\left[\frac{\hat{\operatorname{det}} \mathcal{M}_{I}}{\operatorname{det} \mathcal{M}_{0}}\right]^{-1} e^{-2 S_{I}} \\
& =\frac{1}{2 \pi}\left(\frac{g}{32}\right)^{p-1} \Gamma(p) e^{-2 S_{I}}
\end{aligned}
$$

The leading contribution to the integral in (3.8) arises not from the bosonic critical point at $\tau_{0}=\beta / 2$, but instead from the tail of the thimble attached to the critical point 


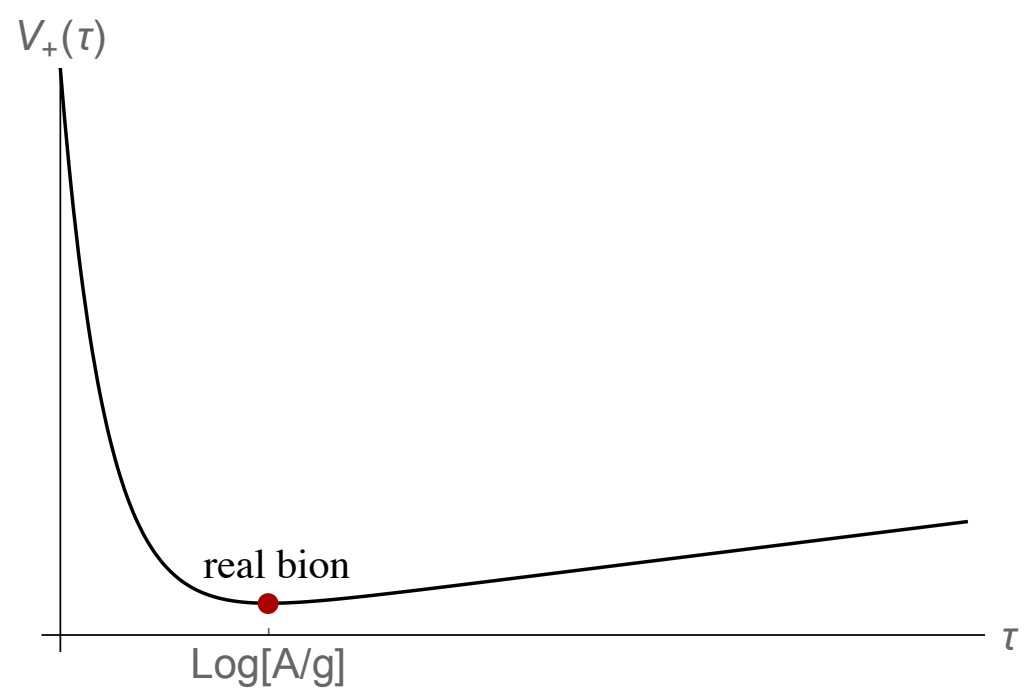

Figure 2. The effective interaction potential $\mathcal{V}_{+}(\tau)$ associated with the $[\mathcal{I I}]$ configuration. The critical value $\tau^{*}=\ln \left(\frac{A}{g p}\right)$ gives the dominant contribution to the $[\mathcal{I} \mathcal{I}]$ amplitude integral in $(3.8)$.

$\tau^{*}=\ln \left(\frac{A}{g p}\right)$. See figure 2 for a sketch of the form of the $[\mathcal{I I}]$ interaction potential. The scale corresponding to this dominant contribution

$$
\tau^{*}=\ln \left(\frac{A}{g p}\right)
$$

is interpreted physically as the separation between the two instantons [12, 23]. In section 3.2 we show the result (3.9), including the pre-exponential terms, matches the contribution of the real bion solution of the quantum potential.

\subsection{2 $[\mathcal{I} \overline{\mathcal{I}}]$ thimble integration}

We now consider the effect of an $[\mathcal{I} \overline{\mathcal{I}}]$ pair. The integration over the quasi-zero mode direction now becomes

$$
[\mathcal{I} \overline{\mathcal{I}}]_{\text {naive }}=\int_{0}^{\beta} d \tau e^{\frac{A}{g}\left(e^{-\tau}+e^{-(\beta-\tau)}\right)} e^{-p \tau}
$$

Here, we have written the integral along the positive real line. This is clearly too naive. The QZM integration should be carried out over the Lefschetz thimble associated with the critical point. The critical point at infinity, $\tau_{0}=\beta / 2$, of the bosonic action is the same as for the $p=0$ theory. The contribution of the vicinity of this critical point is

$$
\int_{\Gamma_{\text {critical point }}} d \tau e^{\frac{A}{g}\left(e^{-\tau}+e^{-(\beta-\tau)}\right)} e^{-p \tau} \approx e^{\frac{2 A}{g}\left(e^{-\beta / 2}\right)} e^{-p \beta / 2},
$$

which vanishes in the limit $\beta \rightarrow \infty$

$$
\lim _{\beta \rightarrow \infty} e^{\frac{2 A}{g}\left(e^{-\beta / 2}\right)} e^{-p \beta / 2}=0 .
$$

As for the $[\mathcal{I I}]$ saddle, this does not mean that the contribution of the thimble vanishes. 


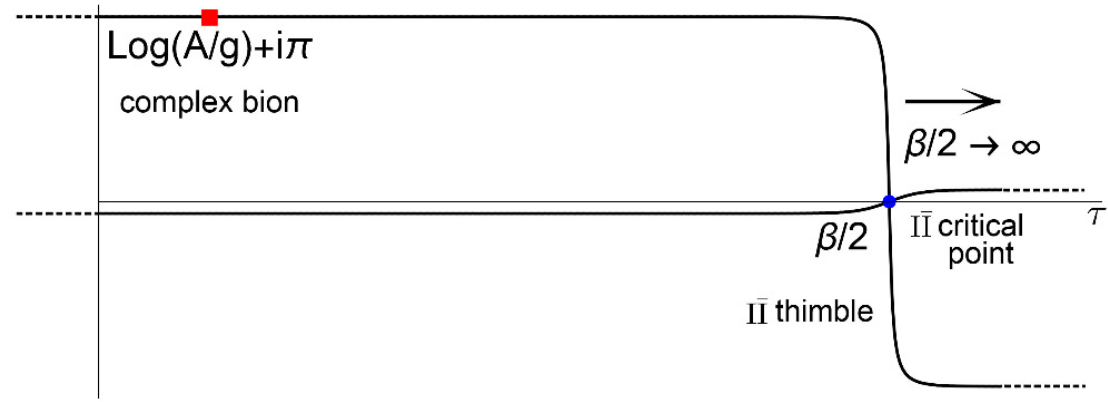

Figure 3. Plot of the Lefschetz thimble attached to the $[\mathcal{I} \overline{\mathcal{I}}]$ critical point at $\tau_{0}=\beta / 2$. As $\beta \rightarrow \infty$, the critical point moves to infinity and its contribution to the thimble integral vanishes. However, the integral receives a non-vanishing contribution from a complex bion configuration located at $\tau_{+}^{*}=\log (A / g)+i \pi$. Compare with figure 2.

The QZM direction is non-Gaussian, and this fact is encoded in the thimble integration. The contour should be deformed along the downward flow direction, as in figure 1. Then the exact integration over the quasi-zero mode thimble $\Gamma_{\mathrm{QZM}}^{\theta=0^{ \pm}}$in the $\beta \rightarrow \infty$ limit results in

$$
I_{-}(p, g) \equiv \int_{\Gamma_{\mathrm{QZM}}^{\theta=0^{ \pm}}} d \tau e^{\frac{A}{g}\left(e^{-\tau}+e^{-(\beta-\tau)}\right)} e^{-p \tau}=e^{ \pm i \pi p}\left(\frac{g}{A}\right)^{p} \Gamma(p) .
$$

The $[\mathcal{I} \overline{\mathcal{I}}]$ amplitude, derived from integrating over this QZM-thimble, is given by

$$
\begin{aligned}
{[\overline{\mathcal{I}}]_{ \pm} } & =I_{-}(p, g) \times[\mathcal{I}]^{2} \\
& =e^{ \pm i \pi p}\left(\frac{g}{A}\right)^{p} \Gamma(p) \times \frac{S_{I}}{2 \pi}\left[\frac{\hat{\operatorname{det}} \mathcal{M}_{I}}{\operatorname{det} \mathcal{M}_{0}}\right]^{-1} e^{-2 S_{I}} \\
& =\frac{1}{2 \pi}\left(\frac{g}{32}\right)^{p-1} \Gamma(p) e^{-2 S_{I}} e^{ \pm i \pi p} .
\end{aligned}
$$

Here, use was made of the fact that the non-zero mode determinant of the $[\mathcal{I} \overline{\mathcal{I}}]$ approximately factorizes.

This result for the $[\mathcal{I} \overline{\mathcal{I}}]$ amplitude has a number of interesting aspects:

- Unlike regular Gaussian saddle points for which critical point gives the dominant contribution, the critical point at infinity and its vicinity do not contribute. This is different from the usual situation in Picard-Lefschetz theory, which by definition has only Gaussian modes because, strictly speaking, the "action" must be a Morse function.

- The integral in (3.14) is dominated by the tail of the Lefschetz thimble in the sense that the main contribution comes from the complex points

$$
\tau_{ \pm}^{*}=\left[\ln \left(\frac{A}{g p}\right) \pm i \pi\right]
$$

where the real part of $\tau_{ \pm}^{*}$ corresponds to the separation between the instanton and anti-instanton constituents [12, 23].

- We show below in section 3.3 that this contribution corresponds to the exact complex bion solution of the quantum modified equations of motion. 


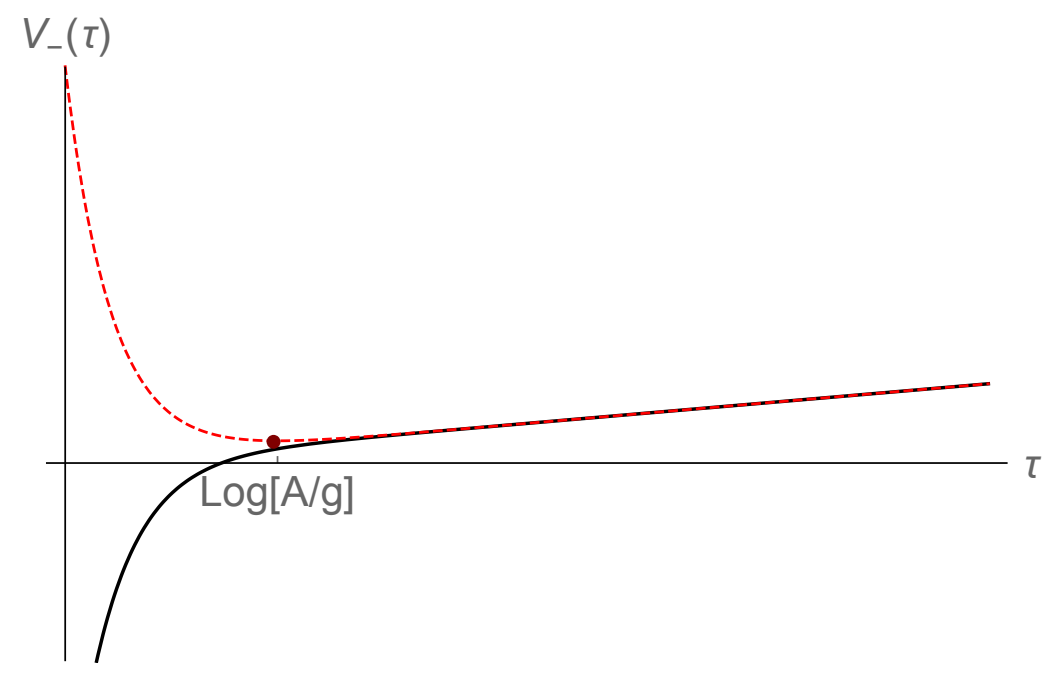

Figure 4. The effective interaction potential $\mathcal{V}_{-}(\tau)$ associated with the $[\mathcal{I} \overline{\mathcal{I}}]$ configuration. For real values of the separation $\tau$, (black-solid curve), this completely attractive potential is the reason why the $[\mathcal{I} \overline{\mathcal{I}}]$ configuration is viewed as unstable in the literature. The red-dashed curve correspond to the effective potential on the thimble. The value $\tau^{*}=\ln \left(\frac{A}{g p}\right)+i \pi$ gives the dominant contribution to the $[\mathcal{I} \overline{\mathcal{I}}]$ amplitude integral in $(3.14)$.

- By expressing the $[\mathcal{I} \overline{\mathcal{I}}]_{ \pm}$amplitude as

$$
[\mathcal{I} \overline{\mathcal{I}}]_{ \pm}=\frac{1}{2 \pi}\left(\frac{g}{32}\right)^{p-1} \Gamma(p) e^{-2 S_{I}}(\cos (\pi p) \pm i \sin (\pi p))
$$

it is easy to see that the two-fold ambiguous part $\sim \pm i \sin (\pi p) \Gamma(p) e^{-2 S_{I}}$ must be related to resurgent cancellations. This part cancels against the ambiguity in the Borel resummation of perturbation theory, as verified in [23, 24].

The unambiguous term $\cos (\pi p)$ is the hidden topological angle (HTA) associated with the bion configuration. The HTA ensures that the complex bion contribution to the ground state energy can be either positive or negative depending on $p$, despite the fact that the Lefschetz thimble belongs to a real critical point at infinity. ${ }^{8}$ In particular, in supersymmetric QM the ground state energy vanishes due to an exact cancellation between the semi-classical contributions of real and complex bions $[12,23]$.

In figure 4 we plot the potential $\mathcal{V}_{-}(\tau)$ for $i$ ) real values of $\tau$ and $i i$ ) for values of $\tau \in \Gamma_{\mathrm{QZM}}$, on the $[\mathcal{I} \overline{\mathcal{I}}]$ QZM thimble. For simplicity, consider the $\beta \rightarrow \infty$ limit. For real values, as discussed in textbooks and reviews, the potential (shown by the black solid curve) is always attractive and the $[\mathcal{I} \overline{\mathcal{I}}]$ configuration is unstable. There is no length scale at which the pair stabilizes. This is the perspective that one obtains by using standard techniques such as real gradient flow or the valley method. On the other hand, on the

\footnotetext{
${ }^{8}$ Recall that the contribution of all real (instanton) configurations to ground state energy is negative semi-definite (in the absence of topological theta-angles or Berry phases accompanied with the instanton amplitudes.). The fact that the complex bion contribution may be positive has very important implications both in QM as well as QFT [22, 54].
} 
QZM-thimble, the exponential (classical) part becomes repulsive as shown in the red dashed curve. The one-loop quantum-induced term leads to an attractive "force", resulting in a stable configuration at $\tau^{*}=\ln \left(\frac{A}{g p}\right)+i \pi$. However, since this latter term is quantum mechanical in origin, the minima are not critical points, but points on the tail of genuine $[\mathcal{I} \overline{\mathcal{I}}]$-thimbles which dominate the integral.

One may think that this description is essentially the analytic continuation of BZJ, $g \rightarrow-g$, which turns the attractive potential to a repulsive one. However, this is not true. In the BZJ prescription, after analytic continuation, the range of integration for $\tau$ is $[0, \infty)$. This range differs from the one appearing on $\Gamma_{\mathrm{QZM}}$. As explained around (2.16), the integration on the BZJ domain induces an exponentially small term $e^{-A / g}$ that would become exponentially large $e^{+A / g}$ once one moves back to the physical theory. This term was dropped in the BZJ analysis without justification. On the other hand, the integration on $\Gamma_{\text {QZM }}$ does not generate this pathological term. As emphasized earlier, in order to obtain this result, one needs to use the complex gradient equation to determine the integration cycle as opposed to the real gradient flow or valley techniques. Once this is done, the analysis of the $[\mathcal{I} \overline{\mathcal{I}}]$ is fundamentally on the same footing as $[\mathcal{I I}]$.

\subsection{Real bion amplitude vs. $[\mathcal{I} \mathcal{I}]$ amplitude}

The semi-classical treatment of the supersymmetric SG system was shown to require the inclusion of the real bion, which is a solution of the one-loop quantum action [12, 23]. The properties of the real bion are reviewed in appendix A.1. The real bion configuration is a solution of the Euclidean equations of motion (3.5) which includes quantum one-loop effects (see (3.2)). In this section we explain the relation between this solution and the $[\mathcal{I I}]$ amplitude (3.9) computed in section 3.1.1, obtained by using the classical action and integrating over $\Gamma_{\mathrm{QZM}}$.

This real bion solution has an exact translational zero mode, the center position of the bion. It also has a parametrically small "soft mode" that will be important below. The amplitude is given by

$$
I_{\text {real bion }}=[\mathcal{R \mathcal { B }}]=J_{t_{c}}\left[\frac{\hat{\operatorname{det}} \mathcal{M}_{\text {real bion }}}{\operatorname{det} \mathcal{M}_{0}}\right]^{-\frac{1}{2}} e^{-S_{\text {real bion }}} .
$$

Here, $J_{t_{c}}=\left[S_{\text {real bion }} /(2 \pi)\right]^{1 / 2}$ is the Jacobian associated with the translational zero mode of the bion, $S_{\text {real bion }}$ is the real bion action, and the determinant is that of the fluctuation operator in the real bion background. We now show that, taking into account the soft mode of the fluctuation operator, the real bion amplitude coincides precisely with the correlated $[\mathcal{I I}]$ amplitude in (3.9). This is a non-trivial result, because the details of the two calculations differ in several significant ways:

- In the $[\mathcal{I I}]$ calculation there are two powers of the Jacobian $J_{t_{c}}$, one for each instanton. In the $[\mathcal{R B}]$ analysis, however, there is only one zero mode and the Jacobian enters only once. Note that the Jacobian is proportional to the square root of the classical action. Since $S_{\text {real bion }} \sim 2 S_{I}$, it is certainly not the case that the Jacobian factors match. 
- For $[\mathcal{I I}]$ there is an integral over the QZM direction, corresponding to the $[\mathcal{I I}]$ separation. The size of the real bion, on the other hand, is fixed by the value of the coupling constant $g$. The existence of a non-Gaussian mode must be encoded in the presence of a parametrically small eigenvalue in the fluctuation operator. It is not immediately obvious that this contribution matches the one arising from the QZM integration.

- The two calculations also differ in the treatment of the classical $[\mathcal{I} \mathcal{I}]$ interaction. In the conventional instanton calculus, the interaction is determined from an ansatz (or an approximate flow equation), and then included in the QZM integral. In the $[\mathcal{R B}]$ analysis $[12,23]$ we observe that the saddle point has action $S_{\text {real bion }}=$ $2 S_{I}-p \log \left(\frac{p g}{32 e}\right)+\ldots$ The logarithmic correction term must combine with the parametrically small eigenvalue of the fluctuation operator to build the integral over the QZM in the $[\mathcal{I I}]$ analysis. We now demonstrate this explicitly.

The real bion fluctuation determinant is computed in appendix A.1. We combine the Jacobian factor, proportional to $\left(S_{\text {saddle }}\right)^{1 / 2}$, with the inverse square root of the determinant (A.14) of the fluctuation operator, to obtain the semi-classical pre-factor:

$$
\sqrt{\frac{S_{\text {real bion }}}{2 \pi}}\left[\frac{\operatorname{det} \mathcal{M}_{\text {real bion }}}{\operatorname{det} \mathcal{M}_{0}}\right]^{-\frac{1}{2}} \approx \frac{1}{2 \pi}\left(\frac{32}{g}\right) \sqrt{\frac{2 \pi}{p}} .
$$

Note that in the weak coupling limit, the relation between the determinant in the real bion (A.14) and the instanton background (2.7) is given by

$$
\frac{\hat{\operatorname{det} \mathcal{M}_{\text {real bion }}}}{\operatorname{det} \mathcal{M}_{0}} \approx \frac{p g}{64}=\frac{p g}{4}\left[\frac{\hat{\operatorname{det} \mathcal{M}_{I}}}{\operatorname{det} \mathcal{M}_{0}}\right]^{2}=\frac{p g}{4}\left[\frac{1}{4}\right]^{2} .
$$

This result proves the existence of one parametrically small eigenvalue in the real bion background. We can view the presence of a small eigenvalue as the result of tunneling between the two minima in the potential of the fluctuation operator, see figure 5. Expressing the bion action in terms of the instanton action, we get

$$
e^{-S_{\text {real bion }}} \sim\left(\frac{g}{32}\right)^{+p}\left(\frac{p}{e}\right)^{+p} e^{-2 S_{I}}
$$

Combining (3.19) and (3.21) the real bion amplitude is obtained as

$$
[\mathcal{R B}]=I_{\text {real bion }}=\frac{1}{2 \pi}\left(\frac{g}{32}\right)^{+p-1}\left(\frac{p}{e}\right)^{+p} \sqrt{\frac{2 \pi}{p}} e^{-2 S_{I}} .
$$

Now compare this real bion result with the $[\mathcal{I I}]$ amplitude in (3.9). We observe that $(p / e)^{p} \sqrt{2 \pi / p}$ is the asymptotic expansion of $\Gamma(p)$ for large $p$, so that the two results agree if we substitute the full expression for its asymptotic form. It is instructive to understand why (3.22) only reproduces the asymptotic form. The crucial observation is that we have treated the soft mode in the real bion analysis as if it is a Gaussian mode.

More specifically, note that the QZM integration (in the $\beta \rightarrow \infty$ limit) is given by

$$
\int_{\Gamma_{\mathrm{QZM}}, \mathrm{exact}} d \tau e^{-\left(\frac{A}{g} e^{-\tau}+p \tau\right)}=\left(\frac{g}{A}\right)^{p} \Gamma(p) .
$$


If we momentarily ignore the fact that the second term in the exponent is a quantum correction, and perform a saddle-point analysis of the effective potential $V(\tau)=\frac{A}{g} e^{-\tau}+p \tau$, we find that the critical point is at $\tau^{*}=\ln \left(\frac{A}{g p}\right)$, which is the size of the real bion. Using a Gaussian approximation, we find

$$
e^{-V\left(\tau_{*}\right)} \int d \tau e^{-\frac{p}{2} \tau^{2}}=\left(\frac{p g}{A e}\right)^{p} \sqrt{\frac{2 \pi}{p}},
$$

which is the leading asymptotic approximation to the exact result given in (3.23). This implies that it is in general not justified to treat the soft mode in the real bion fluctuation operator in the Gaussian approximation. If the soft mode of the real bion was treated consistently, the result would agree with (3.9).

\subsection{Complex bion amplitude versus $[\mathcal{I} \overline{\mathcal{I}}]$ amplitude}

The SG system also has exact complex bion solutions [12, 23]. The complex bion configuration $[\mathcal{C B}]$ is an exact solution of the Euclidean equations of motions (3.5) derived from the quantum potential (3.2). In this section we explain its relation to the $[\mathcal{I} \overline{\mathcal{I}}]$ amplitude (3.15) computed in section 3.1.2, obtained using just the bosonic action. Additional novel features appear in the case of $[\mathcal{I} \overline{\mathcal{I}}]$ pairs, beyond those discussed in the previous section for the case of the $[\mathcal{I I}]$ amplitude.

- In the $[\mathcal{I} \overline{\mathcal{I}}]_{ \pm}$analysis the HTA arises from the integration over the QZM-cycle. In the $[\mathcal{C B}]_{ \pm}$calculation the HTA appears as the imaginary part of the action.

- In instanton calculus the Jacobian and the fluctuation determinant are manifestly real. In the complex bion analysis, the Jacobian and fluctuation determinant could potentially modify the HTA arising from the classical action. We will see that this is not the case. The complex action in the Jacobian cancels exactly against a similar factor that appears in the GY method of calculating the determinant $\left[\hat{\operatorname{det}} \mathcal{M}_{\text {complex bion }}\right]^{-1 / 2}$.

The complex bion fluctuation determinant is computed in appendix A.2. We obtain the counterpart of the real bion result (3.22), in the weak coupling limit, as

$$
[\mathcal{C B}]_{ \pm}=\frac{1}{2 \pi}\left(\frac{g}{32}\right)^{+p-1}\left(\frac{p}{e}\right)^{+p} \sqrt{\frac{2 \pi}{p}} e^{-2 S_{I}} e^{ \pm i p \pi}=[\mathcal{R B}] e^{ \pm i p \pi}
$$

This result shows that, up to the hidden topological phase, the one-loop amplitudes for the complex and the real bion cases are equal. Crucially, the complex bion is a point lying on the QZM-thimble of the instanton/anti-instanton critical point at infinity: $[\mathcal{I} \overline{\mathcal{I}}]_{ \pm}$. This explains why the two types of computations are related. This thimble is shown in figure 3, and the complex bion configuration corresponds to a complex separation between the instanton and anti-instanton constituents given by

$$
\tau_{ \pm}^{*}=\left(\ln \left(\frac{A}{g p}\right) \pm i \pi\right)
$$

Correcting for the soft mode that arises from the complex bion analysis, one finds precisely the $[\mathcal{I} \overline{\mathcal{I}}]_{ \pm}$amplitude in (3.15), obtained over the QZM-thimble of the critical point at infinity. 


\section{Conclusions}

In this work we clarified the treatment of multi-instanton contributions in the quantum mechanical path integral. Our main findings are:

1) Generic multi-instanton contributions correspond to critical points at infinity, and the correct way to compute them is to calculate the integral over the associated Lefschetz thimble. Typically, the main contribution comes not from the saddle point or its vicinity, but from a non-Gaussian integral on the associated complexified quasizero mode manifold.

2) In the theories with fermions the leading contribution is captured by an exact solution of the equations of motion in the quantum modified potential. We showed how to compute the fluctuation operators around these solutions. The fluctuation operator encodes the exact asymptotic behavior of the thimble integral. In order to reproduce the full result the non-Gaussian mode has to be treated exactly, not just in a Gaussian approximation.

3) We demonstrated that the fluctuation operator can be computed for both real and complex (singular) bion solutions. The result has the correct form to match the thimble integration. In particular, the cancellations between real bions and complex singular bions that are required by supersymmetry are preserved when fluctuations are included.

There are some obvious directions in which the present study can be extended. Within quantum mechanics, it will be interesting to study the thimbles that appear in correlated multi-instanton events beyond second order. In quantum field theory, there are applications to the non-BPS multi-instanton and multi-monopole amplitudes discussed in [55-60].

\section{Acknowledgments}

M.̈̈. thanks Nick Manton for discussions. We thank the KITP at UC Santa Barbara for its hospitality during the program "Resurgent Asymptotics in Physics and Mathematics" where some of this work was done. Research at KITP is supported by the National Science Foundation under Grant No. NSF PHY-1125915. This material is based upon work supported by the U.S. Department of Energy, Office of Science, Office of High Energy Physics under Award Number DE-SC0010339 (GD), and by the U.S. Department of Energy, Office of Science, Office of Nuclear Physics under Award Number DE-FG0203ER41260 (AB,TSc,MÜ).

\section{A Fluctuation determinants}

In this appendix we record the computations of the fluctuation determinants around the bion configurations discussed in section 3 . We compute the determinant with the zero mode removed using an appropriate variant $[47,49,51,52]$ of the general determinant method 
of Gel'fand-Yaglom [44-50]. In this approach, the determinant with zero mode removed is expressed entirely in terms of the associated classical action and the asymptotic data of the zero mode as

$$
\frac{\hat{\operatorname{det}} \mathcal{M}}{\operatorname{det} \mathcal{M}_{0}}=\frac{g}{2 \omega A_{+} A_{-}} S_{\text {saddle }}
$$

where the fluctuation operator about a $\mathrm{SG}$ saddle solution is given as

$$
\begin{aligned}
\mathcal{M}_{\text {saddle }} & =-\frac{d^{2}}{d t^{2}}+V^{\prime \prime}\left(z_{\text {saddle }}(t)\right) \\
& =-\frac{d^{2}}{d t^{2}}+\cos \left(z_{\text {saddle }}(t)\right)+\frac{p g}{8} \cos \left(\frac{1}{2} z_{\text {saddle }}(t)\right),
\end{aligned}
$$

and the zero mode is the derivative of the classical saddle solution, with asymptotics

$$
\dot{z}(t) \approx A_{ \pm} e^{\mp \omega t} \quad \text { for } t \rightarrow \pm \infty .
$$

The determinant is normalized by the determinant of the corresponding free operator with frequency $\omega, \mathcal{M}_{0}=-\frac{d^{2}}{d t^{2}}+\omega^{2}$. For fluctuation operators about instantons, this is all very well known. For the SG system, the corresponding instanton zero mode is

$$
\dot{x}_{I}=2 \operatorname{sech}(t) \sim 4 e^{\mp t} \quad, \quad t \rightarrow \pm \infty,
$$

from which the result quoted in (2.7) follows. We now apply the general formula (A.1) to the results of $[12,23]$, to compute the fluctuation determinants for the various bion solutions of the SG system.

\section{A.1 Fluctuation determinant for real bion saddle}

After integrating out $p$ flavors of fermions, the SG bosonic quantum potential is

$$
V(x)=\frac{1}{2}\left(W^{\prime}\right)^{2}+\frac{1}{2} p g W^{\prime \prime}=2 \sin ^{2}\left(\frac{x}{2}\right)-\frac{1}{2} p g \cos \left(\frac{x}{2}\right) .
$$

The complexified classical equations of motion are

$$
\ddot{z}=\sin (z)+\frac{1}{4} g p \sin \left(\frac{z}{2}\right) .
$$

The equations of motion (A.6) have a real bion solution [12, 23], which has the form of a correlated instanton-instanton pair,

$$
z_{\text {real bion }}(t)=4\left(\arctan \left(\exp \left[\omega\left(t-t_{c}-t_{0}\right)\right]\right)+\arctan \left(\exp \left[\omega\left(t-t_{c}+t_{0}\right)\right]\right)\right),
$$

where the frequency $\omega=\omega_{\text {real bion }}$ is

$$
\omega_{\text {real bion }}=\sqrt{1+\frac{p g}{8}} .
$$

The translational collective coordinate of the bion is $t_{c}$, and $t_{0}$ is the characteristic separation scale

$$
t_{0}^{\text {real bion }} \equiv \frac{1}{\omega_{\text {real bion }}} \ln \left[\sqrt{\frac{8}{p g}}\left(1+\omega_{\text {real bion }}\right)\right] \approx \frac{1}{2} \ln \left(\frac{32}{p g}\right)+\ldots
$$




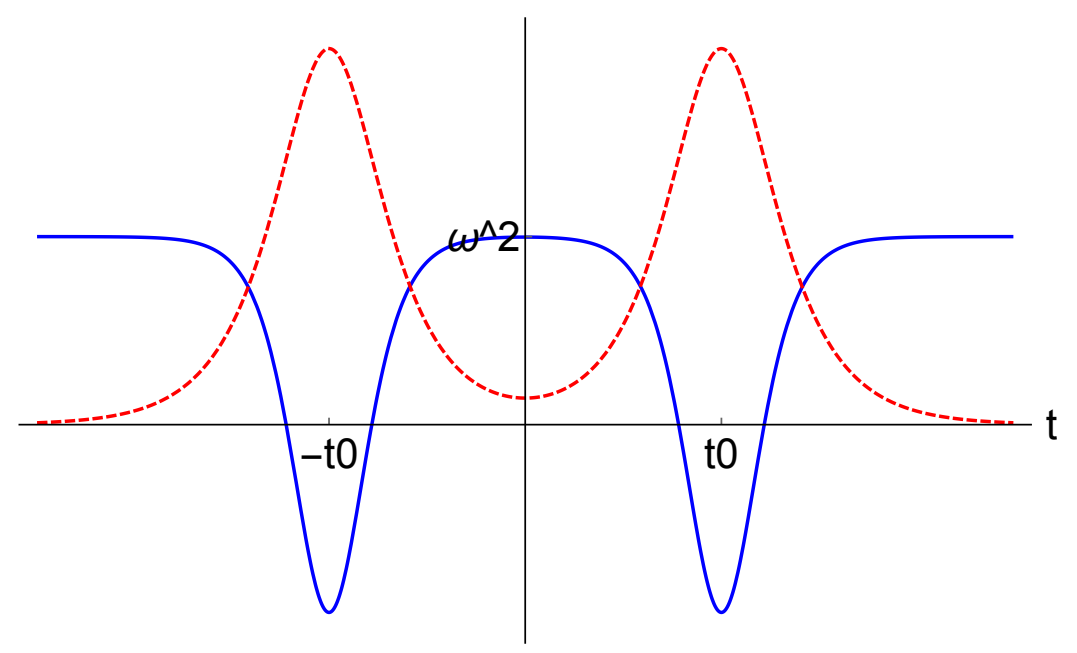

Figure 5. Plot of the fluctuation potential in (A.11) for the SG real bion solution (solid blue curve), together with the zero mode (dashed red curve) in (A.12). Note the double well structure of the fluctuation potential, and the symmetric nature of the zero mode solution, characteristic of a real bion solution, leading to a positive fluctuation determinant.

The action of the SG real bion solution is

$$
\begin{aligned}
S_{\text {real bion }} & =\frac{16}{g} \sqrt{1+\frac{g p}{8}}+2 p \operatorname{arctanh}\left(\frac{1}{\sqrt{1+\frac{p g}{8}}}\right) \\
& =\frac{16}{g}+p \ln \left(\frac{32 e}{p g}\right)+\ldots
\end{aligned}
$$

The fluctuation operator about the SG real bion solution is

$$
\mathcal{M}_{\text {real bion }}=-\frac{d^{2}}{d t^{2}}+\cos \left(z_{\text {real bion }}(t)\right)+\frac{p g}{8} \cos \left(\frac{1}{2} z_{\text {real bion }}(t)\right) .
$$

The form of this fluctuation potential is plotted in figure 5 .

The fluctuation operator in (A.11) has an exact zero mode, associated with translation symmetry

$$
\begin{aligned}
\phi_{\text {zero mode }}^{\text {real bion }}(t) & =\dot{z}_{\text {real bion }}(t) \\
& =2 \omega\left(\operatorname{sech}\left(\omega\left(t-t_{c}-t_{0}\right)\right)+\operatorname{sech}\left(\omega\left(t-t_{c}+t_{0}\right)\right)\right),
\end{aligned}
$$

where $\omega \equiv \omega_{\text {real bion }}$, and $t_{0} \equiv t_{0}^{\text {real bion }}$. This zero mode (A.12) is also plotted in figure 5 . Note the double-well structure of the fluctuation potential, distinct from the familiar singlewell structure of the fluctuation potential (2.6) for the instanton solution. Also note the symmetric form of the real bion zero mode, as expected for the lowest mode of a nonnegative fluctuation operator (contrast with the anti-symmetric zero mode for the bounce, in figure 6). 
To compute the real bion fluctuation determinant we need the asymptotic values of the zero mode as normalized in (A.12). For the SG real bion solution

$$
\phi_{\text {zero mode }}^{\text {real bion }}(t) \sim 4 \sqrt{\frac{32}{p g}} \omega_{\text {real bion }}^{2} e^{\mp \omega_{\text {real bion }} t} \quad, \quad t \rightarrow \pm \infty .
$$

Thus, from the general formula (A.1), the determinant of the fluctuation operator with zero mode removed, relative to that of the corresponding free operator, is given by

$$
\frac{\hat{\operatorname{det}} \mathcal{M}_{\text {real bion }}}{\operatorname{det} \mathcal{M}_{0}} \approx \frac{p g}{64} \text {. }
$$

\section{A.2 Fluctuation determinants for real bounce and complex bion saddles}

We first recall the real bounce solution, because as discussed in detail in $[12,23]$, the complex bion solutions are obtained from the real bounce by the analytic continuation $p \rightarrow-p$. We thus compute the fluctuation determinants from the analytic continuation of the corresponding real bounce fluctuation determinant, as all steps of the computation have a well-defined analytic continuation. We will see that this procedure leads to results that agree perfectly with the physical picture developed in this paper.

The equations of motion (A.6) have a real bounce solution, with the form of a correlated instanton/anti-instanton pair

$$
z_{\text {bounce }}(t)=2 \pi+4\left[\arctan \left(\exp \left[\omega\left(t-t_{c}-t_{0}\right)\right]\right)-\arctan \left(\exp \left[\omega\left(t-t_{c}+t_{0}\right)\right]\right)\right]
$$

The frequency $\omega$ in (A.15) is

$$
\omega_{\text {bounce }}=\sqrt{1-\frac{p g}{8}}
$$

and the characteristic separation scale $t_{0}$ in (A.15) is

$$
t_{0}^{\text {bounce }} \equiv \frac{1}{\omega_{\text {bounce }}} \ln \left[\sqrt{\frac{8}{p g}}\left(1+\omega_{\text {bounce }}\right)\right] \approx \frac{1}{2} \ln \left(\frac{32}{p g}\right)+\ldots
$$

The action of the SG bounce solution is

$$
\begin{aligned}
S_{\text {bounce }} & =\frac{16}{g} \sqrt{1-\frac{g p}{8}}-2 p \operatorname{arctanh}\left[\sqrt{1-\frac{g p}{8}}\right] \\
& =\frac{16}{g}-p \ln \left(\frac{32 e}{p g}\right)+\ldots
\end{aligned}
$$

The fluctuation operator about this SG bounce solution is

$$
\mathcal{M}_{\text {bounce }}=-\frac{d^{2}}{d t^{2}}+\cos \left(z_{\text {bounce }}(t)\right)+\frac{p g}{8} \cos \left(\frac{1}{2} z_{\text {bounce }}(t)\right) .
$$

The form of this fluctuation potential is plotted in figure 6 . 


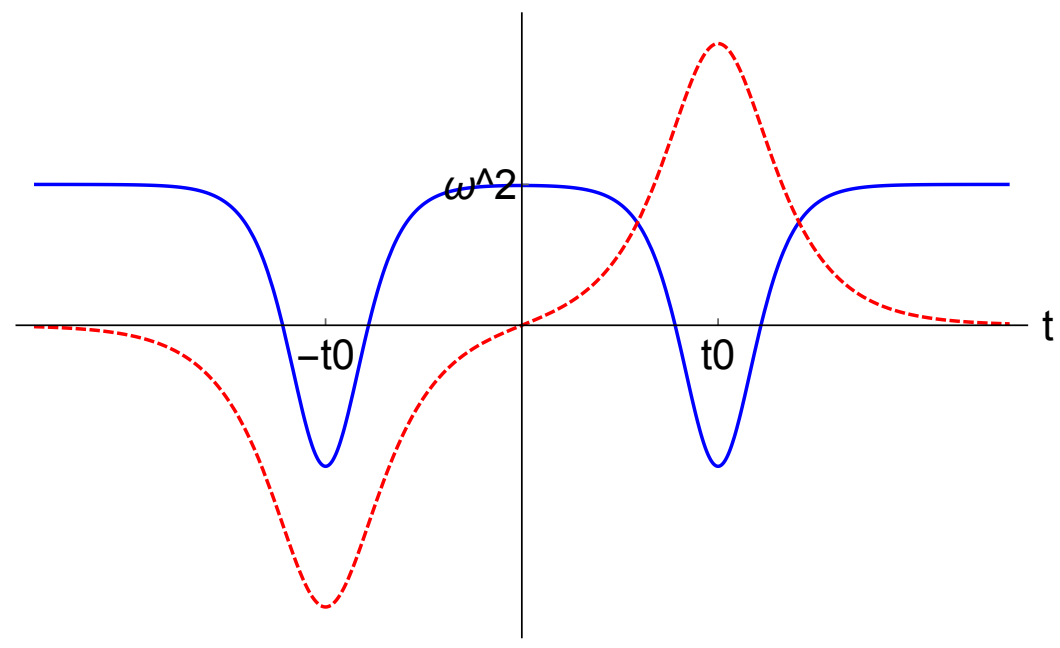

Figure 6. Plot of the fluctuation potential in (A.19) for the SG bounce solution (solid blue curve), together with the zero mode (dashed red curve) in (A.20). Note the double-well structure of the fluctuation potential, and the anti-symmetric nature of the zero mode solution, characteristic of a bounce solution, leading to a negative fluctuation determinant.

This fluctuation operator in (A.19) has an exact zero mode, associated with translation symmetry

$$
\begin{aligned}
\phi_{\text {zero mode }}^{\text {bounce }}(t) & =\dot{z}_{\text {bounce }}(t) \\
& =2 \omega\left(\operatorname{sech}\left(\omega\left(t-t_{c}-t_{0}\right)\right)-\operatorname{sech}\left(\omega\left(t-t_{c}+t_{0}\right)\right)\right),
\end{aligned}
$$

where $\omega \equiv \omega_{\text {bounce }}$, and $t_{0} \equiv t_{0}^{\text {bounce }}$. This SG bounce zero mode (A.20) is also plotted in figure 6. Note the double-well structure, distinct from the familiar single-well structure of the fluctuations about the instanton solution. Also note the anti-symmetric form of the bounce zero mode, indicating the existence of a negative mode, as expected for a bounce solution (contrast with the symmetric zero mode for the real bion in figure 5).

To compute the fluctuation determinant we need the asymptotic values of the zero mode, as normalized in (A.20). For this SG bounce solution

$$
\phi_{\text {zero mode }}^{\text {bounce }}(t) \sim \pm 8 \sqrt{\frac{8}{p g}} \omega_{\text {bounce }}^{2} e^{\mp \omega_{\text {bounce }} t} \quad, \quad t \rightarrow \pm \infty .
$$

Thus, from the general formula (A.1), the determinant of the bounce fluctuation operator with zero mode removed, relative to that of the corresponding free operator, is given by

$$
\frac{\hat{\operatorname{det}} \mathcal{M}_{\text {bounce }}}{\operatorname{det} \mathcal{M}_{0}} \approx-\frac{p g}{64} \text {. }
$$

The supersymmetric SG system also has a complex bion solution, from the true vacuum critical point to (a complex conjugate pair of) complex turning points. As discussed in detail in $[12,23]$, this solution is obtained by implementing the analytic continuation $p \rightarrow$ $e^{ \pm i \pi} p$ in the (real) bounce solution. Thus, by an analogous computation, the corresponding fluctuation determinant is

$$
\frac{\hat{\operatorname{det}} \mathcal{M}_{\text {complex bion }}}{\operatorname{det} \mathcal{M}_{0}} \approx \frac{p g}{64} \text {. }
$$


Open Access. This article is distributed under the terms of the Creative Commons Attribution License (CC-BY 4.0), which permits any use, distribution and reproduction in any medium, provided the original author(s) and source are credited.

\section{References}

[1] S.R. Coleman, Aspects of Symmetry, Cambridge University Press (1979).

[2] J. Zinn-Justin, Quantum field theory and critical phenomena, Int. Ser. Monogr. Phys. 113 (2002) 1 [INSPIRE].

[3] E.B. Bogomolny, Calculation of instanton-anti-instanton contributions in quantum mechanics, Phys. Lett. B 91 (1980) 431 [INSPIRE].

[4] J. Zinn-Justin, Multi-Instanton Contributions in Quantum Mechanics, Nucl. Phys. B 192 (1981) 125 [INSPIRE].

[5] A. Cherman, D. Dorigoni and M. Ünsal, Decoding perturbation theory using resurgence: Stokes phenomena, new saddle points and Lefschetz thimbles, JHEP 10 (2015) 056 [arXiv: 1403.1277] [INSPIRE].

[6] G.V. Dunne and M. Ünsal, Resurgence and Trans-series in Quantum Field Theory: The CP(N-1) Model, JHEP 11 (2012) 170 [arXiv:1210.2423] [INSPIRE].

[7] A. Cherman, D. Dorigoni, G.V. Dunne and M. Ünsal, Resurgence in Quantum Field Theory: Nonperturbative Effects in the Principal Chiral Model, Phys. Rev. Lett. 112 (2014) 021601 [arXiv: 1308.0127] [INSPIRE].

[8] T. Misumi, M. Nitta and N. Sakai, Resurgence in sine-Gordon quantum mechanics: Exact agreement between multi-instantons and uniform WKB, JHEP 09 (2015) 157 [arXiv: 1507.00408] [INSPIRE].

[9] G. Basar, G.V. Dunne and M. Ünsal, Resurgence theory, ghost-instantons and analytic continuation of path integrals, JHEP 10 (2013) 041 [arXiv: 1308.1108] [INSPIRE].

[10] P.C. Argyres and M. Ünsal, The semi-classical expansion and resurgence in gauge theories: new perturbative, instanton, bion and renormalon effects, JHEP 08 (2012) 063 [arXiv: 1206.1890] [INSPIRE].

[11] M.M. Anber and T. Sulejmanpasic, The renormalon diagram in gauge theories on $\mathbb{R} 3 \times \mathbb{S} 1$, JHEP 01 (2015) 139 [arXiv: 1410.0121] [INSPIRE].

[12] A. Behtash, G.V. Dunne, T. Schäfer, T. Sulejmanpasic and M. Ünsal, Toward Picard-Lefschetz Theory of Path Integrals, Complex Saddles and Resurgence, arXiv: 1510.03435 [INSPIRE].

[13] G.V. Dunne and M. Ünsal, New Nonperturbative Methods in Quantum Field Theory: From Large-N Orbifold Equivalence to Bions and Resurgence, Ann. Rev. Nucl. Part. Sci. 66 (2016) 245 [arXiv : 1601.03414] [INSPIRE].

[14] T. Sulejmanpasic and M. Ünsal, Aspects of perturbation theory in quantum mechanics: The BenderWu Mathematica ${ }^{\circledR}$ package, Comput. Phys. Commun. 228 (2018) 273 [arXiv: 1608.08256] [INSPIRE].

[15] M. Kontsevich, On non-perturbative quantization, fukaya categories and resurgence, talk at Simons Center, (2015) [http://scgp.stonybrook.edu/video_portal/video.php?id=2183]. 
[16] M. Kontsevich, Resurgence from the path integral perspective, talk at Perimeter Institute, (2012) [https://www.perimeterinstitute.ca/videos/resurgence-path-integral-perspective].

[17] E. Witten, A New Look At The Path Integral Of Quantum Mechanics, arXiv:1009.6032 [INSPIRE].

[18] E. Witten, Analytic Continuation Of Chern-Simons Theory, AMS/IP Stud. Adv. Math. 50 (2011) 347 [arXiv: 1001.2933] [INSPIRE].

[19] D. Harlow, J. Maltz and E. Witten, Analytic Continuation of Liouville Theory, JHEP 12 (2011) 071 [arXiv: 1108.4417] [INSPIRE].

[20] A. Behtash, E. Poppitz, T. Sulejmanpasic and M. Ünsal, The curious incident of multi-instantons and the necessity of Lefschetz thimbles, JHEP 11 (2015) 175 [arXiv: 1507.04063] [INSPIRE].

[21] A. Behtash, More on Homological Supersymmetric Quantum Mechanics, Phys. Rev. D 97 (2018) 065002 [arXiv:1703.00511] [INSPIRE].

[22] A. Behtash, T. Sulejmanpasic, T. Schäfer and M. Ünsal, Hidden topological angles and Lefschetz thimbles, Phys. Rev. Lett. 115 (2015) 041601 [arXiv: 1502.06624] [INSPIRE].

[23] A. Behtash, G.V. Dunne, T. Schäfer, T. Sulejmanpasic and M. Ünsal, Complexified path integrals, exact saddles and supersymmetry, Phys. Rev. Lett. 116 (2016) 011601 [arXiv: 1510.00978] [INSPIRE].

[24] C. Kozçaz, T. Sulejmanpasic, Y. Tanizaki and M. Ünsal, Cheshire Cat resurgence, Self-resurgence and Quasi-Exact Solvable Systems, arXiv:1609.06198 [INSPIRE].

[25] T. Fujimori, S. Kamata, T. Misumi, M. Nitta and N. Sakai, Nonperturbative contributions from complexified solutions in $\mathbb{C} P^{N-1}$ models, Phys. Rev. D 94 (2016) 105002 [arXiv: 1607.04205] [INSPIRE].

[26] D. Dorigoni and P. Glass, The grin of Cheshire cat resurgence from supersymmetric localization, SciPost Phys. 4 (2018) 012 [arXiv:1711.04802] [INSPIRE].

[27] N. Nekrasov, Tying up instantons with anti-instantons, arXiv:1802.04202.

[28] M.V. Fedoryuk, The saddle-point method, Izdat. Nauka, Moscow, MR 58:22580 (1977).

[29] T. Schäfer and E.V. Shuryak, Instantons in QCD, Rev. Mod. Phys. 70 (1998) 323 [hep-ph/9610451] [INSPIRE].

[30] F. Pham, Vanishing homologies and the $n$ variable saddlepoint method, Proc. Symp. Pure Math 2 (1983) 319.

[31] V.I. Arnold, S.M. Gusein-Zade and A.N. Varchenko, Singularities of Differentiable Maps, Volume 1, Birkhäuser Basel (2012).

[32] E. Witten, Constraints on Supersymmetry Breaking, Nucl. Phys. B 202 (1982) 253 [INSPIRE].

[33] M.A. Shifman, Beginning supersymmetry (supersymmetry in quantum mechanics), in ITEP lectures on particle physics and field theory, vol. 1, pp. 301-344, World Scientific, Singapore (1995) [INSPIRE].

[34] I.I. Balitsky and A.V. Yung, Instanton Molecular Vacuum in $N=1$ Supersymmetric Quantum Mechanics, Nucl. Phys. B 274 (1986) 475 [INSPIRE]. 
[35] P.V. Buividovich, G.V. Dunne and S.N. Valgushev, Complex Path Integrals and Saddles in Two-Dimensional Gauge Theory, Phys. Rev. Lett. 116 (2016) 132001 [arXiv:1512.09021] [INSPIRE].

[36] M. Serone, G. Spada and G. Villadoro, Instantons from Perturbation Theory, Phys. Rev. D 96 (2017) 021701 [arXiv:1612.04376] [INSPIRE].

[37] M. Serone, G. Spada and G. Villadoro, The Power of Perturbation Theory, JHEP 05 (2017) 056 [arXiv: 1702.04148] [INSPIRE].

[38] G.V. Dunne, T. Sulejmanpasic and M. Ünsal, Thimbles and Resurgence in the Triple-well System, work in progress.

[39] E. Brézin, J.C. Le Guillou and J. Zinn-Justin, Perturbation Theory at Large Order. 2. Role of the Vacuum Instability, Phys. Rev. D 15 (1977) 1558 [INSPIRE].

[40] R. Balian, G. Parisi and A. Voros, Quartic Oscillator, in Feynman Path Integrals. Proceedings of the International Colloquium held in Marseille, May 1978, pp. 337-360 (1978).

[41] J.L. Richard and A. Rouet, Complex Saddle Points Versus Dilute Gas Approximation in the Double Well Anharmonic Oscillator, Nucl. Phys. B 185 (1981) 47 [InSPIRE].

[42] A. Lapedes and E. Mottola, Complex Path Integrals and Finite Temperature, Nucl. Phys. B 203 (1982) 58 [INSPIRE].

[43] P.A. Millard, Complex Classical Paths and the One-dimensional sine-Gordon System, Nucl. Phys. B 259 (1985) 266 [INSPIRE].

[44] I.M. Gelfand and A.M. Yaglom, Integration in functional spaces and it applications in quantum physics, J. Math. Phys. 1 (1960) 48 [InSPIRE].

[45] S.R. Coleman, The Fate of the False Vacuum. 1. Semiclassical Theory, Phys. Rev. D 15 (1977) 2929 [Erratum ibid. D 16 (1977) 1248] [INSPIRE].

[46] C.G. Callan Jr. and S.R. Coleman, The Fate of the False Vacuum. 2. First Quantum Corrections, Phys. Rev. D 16 (1977) 1762 [InSPIRE].

[47] K. Kirsten, Spectral functions in mathematics and physics, Chapman and Hall/CRC (2001).

[48] H. Kleinert, Path Integrals in Quantum Mechanics, Statistics, Polymer Physics, and Financial Markets, World Scientific (2006).

[49] G.V. Dunne, Functional determinants in quantum field theory, J. Phys. A 41 (2008) 304006 [arXiv:0711.1178] [INSPIRE].

[50] M. Marino, Instantons and Large N, Cambridge University Press (2015).

[51] A.J. McKane and M.B. Tarlie, Regularization of functional determinants using boundary perturbations, J. Phys. A 28 (1995) 6931 [cond-mat/9509126] [INSPIRE].

[52] G.V. Dunne and H. Min, Beyond the thin-wall approximation: Precise numerical computation of prefactors in false vacuum decay, Phys. Rev. D 72 (2005) 125004 [hep-th/0511156] [INSPIRE].

[53] S. Friedli and Y. Velenik, Statistical Mechanics of Lattice Systems: A Concrete Mathematical Introduction, Cambridge University Press (2017),

[54] G.V. Dunne and M. Ünsal, Deconstructing zero: resurgence, supersymmetry and complex saddles, JHEP 12 (2016) 002 [arXiv: 1609.05770] [INSPIRE]. 
[55] M. Ünsal, Magnetic bion condensation: A New mechanism of confinement and mass gap in four dimensions, Phys. Rev. D 80 (2009) 065001 [arXiv:0709.3269] [INSPIRE].

[56] E. Poppitz, T. Schäfer and M. Ünsal, Universal mechanism of (semi-classical) deconfinement and theta-dependence for all simple groups, JHEP 03 (2013) 087 [arXiv:1212.1238] [INSPIRE].

[57] R. Dabrowski and G.V. Dunne, Fractionalized Non-Self-Dual Solutions in the CP(N-1) Model, Phys. Rev. D 88 (2013) 025020 [arXiv:1306.0921] [InSPIRE].

[58] M. Nitta, Fractional instantons and bions in the $O(N)$ model with twisted boundary conditions, JHEP 03 (2015) 108 [arXiv: 1412.7681] [INSPIRE].

[59] Y. Liu, E. Shuryak and I. Zahed, Confining dyon-antidyon Coulomb liquid model. I., Phys. Rev. D 92 (2015) 085006 [arXiv:1503.03058] [InSPIRE].

[60] M. Nitta, Fractional instantons and bions in the principal chiral model on $\mathbb{R}^{2} \times S^{1}$ with twisted boundary conditions, JHEP 08 (2015) 063 [arXiv:1503.06336] [INSPIRE]. 